\title{
Formación Profesional: Requerimientos profesionales y ocupaciones laborales de la CNO-11
}

\section{Vocational Training: Professional requirements and labour occupations of the NCO-11}

\author{
Francisco del Cerro Velázquez \\ Universidad de Murcia. Murcia, España \\ fcerro@um.es \\ Francisco Javier Ramón Cano \\ Universidad de Murcia. Murcia, España \\ jramon@um.es
}

\begin{abstract}
Resumen
Para la estrategia sobre orientación profesional referida a la cuestión de valorar de modo realista la concordancia entre las capacidades e intereses individuales y las exigencias objetivas de las profesiones deseadas, resulta de especial interés las posibilidades que ofrece el análisis de la ocupación laboral cuando se consigue enlazar dicha variable con los títulos de formación profesional y los certificados de profesionalidad $\mathrm{y}$, a su vez, con los requerimientos profesionales. En este trabajo se conjuga el estudio de los siguientes factores de información: la Guía de Valoración Profesional del Instituto Nacional de la Seguridad Social ( $3^{\text {a }}$ edición año 2014), la Clasificación Nacional de Ocupaciones 2011 (CNO-11), las cualificaciones profesionales del Catálogo Nacional de las Cualificaciones Profesionales (CNCP) y los planes de estudio de los títulos de formación profesional y de los certificados de profesionalidad publicados hasta julio del año 2016. Los resultados obtenidos son una amplia relación del binomio formación profesional-ocupación laboral según la CNO-11. El diseño planteado no solo es interesante a nivel estadístico, sino que, además, permite identificar, entre otras, las condiciones psicofísicas ligadas a situaciones de seguridad o salud de los títulos de formación profesional encaminados a desempeñar las ocupaciones laborales más frecuentes vinculadas a dichos estudios.

Palabras clave: orientación profesional, ocupación laboral, formación profesional, cualificación profesional, CNO-11
\end{abstract}

\begin{abstract}
It is of special interest the possibilities offered by the analysis of employment occupation when linking is achieved said variable with the titles of vocational training and certificates of professionalism and, in turn, with professional requirements, for the strategy on professional guidance referred to the issue of realistically assessing the agreement between individual skills and interests and the objective requirements of the desired professions. In this work is combined the study of the following information factors: Professional Valoration Guide of the National Social Security Institute (3rd edition, 2014), National Classification of Occupations 2011 (NCO-11), National Catalog of Professional Qualifications (NCPQ) and the curricula of Vocational training and Certificates of professionalism published up to July of 2016. The results obtained are a broad relationship of the binomial professional trainingoccupation according to the CNO-11. The proposed design is not only interesting at the statistical level, but also allows identifying, among others, psychophysical conditions linked to safety or health situations of vocational training titles aimed at performing the most frequent occupations related to these studies.
\end{abstract}

Keywords: vocational guidance, labour occupation, vocational training, professional qualification, NCO-11 


\section{Introducción}

En España, en mayor o menor medida hay un desajuste entre la formación en competencias que se recibe en el sistema educativo de la formación profesional y el empleo que se desarrolla (Del Cerro Velázquez, F. y Ramón Cano, F.J., 2017). El autor Amor Bravo (2014) especialista en formación, cualificaciones y políticas activas de empleo propugnaba que los motivos que explican el desajuste entre oferta y demanda de cualificación son debidos a un deficiente funcionamiento de los mecanismos de información y orientación del sistema de cualificaciones, además de una limitada difusión de la información sobre salidas laborales. Asimismo, propone una necesaria potenciación de los Servicios Públicos de Empleo (SEPE) para el uso de las cualificaciones como herramienta de intermediación laboral, y para la orientación, tanto hacia el reconocimiento de competencias profesionales como de la formación.

En los últimos años, la cuestión del desajuste de las cualificaciones y el empleo ha recibido especial atención en las economías desarrolladas, así como consecuencia de la crisis económica, a juicio de algunos economistas laborales el desajuste entre la oferta y la demanda de cualificaciones significa que las personas desempleadas necesitarán más tiempo para encontrar un nuevo puesto de trabajo (Theo Sparreboom, comunicación personal, 4 de febrero de 2013). Las enseñanzas de formación profesional reglada (en el aula) persiguen desarrollar al máximo las capacidades de las personas, tanto para la adquisición de las cualificaciones profesionales como para su aplicación al mercado laboral, tremendamente cambiante, que exige capacidad de adaptación y aprendizaje en un mundo expuesto a cambios muy pronunciados y a un proceso de globalización (Mira Lema, 2003). Otros autores como Echeverría, B. (2008) argumentan que las cualidades, aptitudes y destrezas personales que se exigen para poder incorporarse al mundo del trabajo y desenvolverse en la profesión se apartan en buena medida de las tradicionalmente demandadas.

Hoy en día, asistimos a un mercado de trabajo de rápidos cambios tecnológicos, normas de regulación mundiales, poblaciones móviles en crecimiento y aparición constante de nuevos empleos (Cedefop, 2015). Uno de los principios que valida la estructura del planteamiento del sistema educativo de formación profesional se basa en la necesidad de que la cualificación profesional de los alumnos que finalizan los estudios de los distintos títulos de Formación Profesional reglada les permita su inserción en las empresas, para desempeñar un puesto de trabajo vinculado a su perfil profesional (Pérez Esparrells y Rahona, 2009). En efecto, los beneficios de obtener un título de formación profesional le supone al trabajador que tenga más probabilidades de mantener su empleo, de promocionar y, sobre todo, de mejorar su salario (Medina y Sanz, 2009).

Planas (2013) sostiene como uno de los retos a los que se deben hacer frente las Administraciones educativas, el hecho de que planifiquen con agilidad para ampliar la oferta de ciclos de la formación profesional a la realidad socioeconómica del territorio de su competencia, a las propias expectativas de los ciudadanos y a las demandas del tejido productivo. Actualmente, existen elevadas tasas de desempleo juvenil, algo preocupante en los países de la Unión Europea y, en particular, en España, por lo que hay un creciente interés por alcanzar un mejor ajuste entre las competencias profesionales y los requerimientos del mercado de trabajo. En los países desarrollados la empleabilidad está

Formación Profesional: Requerimientos profesionales y ocupaciones laborales de la CNO-11. Francisco del Cerro Velázquez y Francisco Javier Ramón Cano.

Página 2 de 13 
en relación con el nivel de formación de la población activa; es decir, que la empleabilidad es siempre superior en la población de mayor nivel de estudios (OCDE, 2014).

El cambio al modelo de cualificaciones profesionales establecido con la Ley Orgánica de las Cualificaciones y de la Formación Profesional (Ley Orgánica, 2002) donde ya ha transcurrido un quinquenio desde su aplicación ha supuesto una novedad relativamente reciente con la finalidad de mejorar la política de empleo y educativa y, en especial, para la formación profesional, ya que está basada en permitir a una persona alcanzar la cualificación profesional tanto por la vía del aprendizaje y la formación (el aula) como por la experiencia laboral acumulada en su trayectoria personal, lo que pone el énfasis profesional en la "competencia" en vez de en el "título", asumiendo que es la competencia la que faculta para hacer bien el trabajo y, por tanto, la que incrementa la empleabilidad de las personas y la productividad y competitividad de las empresas.

La Ley Orgánica 5/2002 de 19 de junio, de las Cualificaciones y de la Formación Profesional, establece el Sistema Nacional de Cualificaciones y Formación Profesional (SNCyFP) como verdadero eje sobre el que gira la construcción del nuevo marco de la formación profesional en España, adecuado al Marco Europeo de Cualificaciones, y con objetivos de vincular la formación con la política de empleo para fomentar la libre circulación de trabajadores (Cano Galán, 2010). Las cualificaciones profesionales definidas como el conjunto de competencias profesionales con significación para el empleo (Art.7.1 Ley Orgánica 2/2002) tienen su propio Catálogo Nacional (Real Decreto 1416/2005), donde se recogen aquellas más significativas susceptibles de reconocimiento y acreditación en España. Al mismo tiempo, se ordena un catálogo modular que recoge la formación asociada a cada una de las unidades de competencia que son el agregado mínimo que integra la cualificación profesional, organizándose este catálogo en módulos formativos para los que se establecen los correspondientes contenidos y duración, así como otros elementos como son las capacidades a obtener y los criterios de evaluación a aplicar. Los catálogos modulares formativos (módulos profesionales) asociados a cualificaciones profesionales constituyen la base para elaborar la oferta formativa de los títulos y los certificados de profesionalidad, sustentados en textos legislativos donde se describen diferentes aspectos que sirven de base para conformar la información precisa para elaborar un perfil profesional.

El sistema actual de formación profesional reconoce los tres primeros niveles de cualificación profesional (de los cinco existentes) de la forma siguiente:

- Nivel 1: Referido a la formación incluida en los ciclos de Formación Profesional Básica o su correspondiente certificado de profesionalidad de nivel uno en el sistema de formación a través de Empleo.

- Nivel 2: Basado en la formación incluida en los ciclos formativos de grado medio de Formación Profesional o su correspondiente certificado de profesionalidad de nivel dos.

- Nivel 3: Establecido en la formación incluida en los ciclos formativos de grado superior de Formación Profesional o su correspondiente certificado de profesionalidad de nivel tres.

Formación Profesional: Requerimientos profesionales y ocupaciones laborales de la CNO-11. Francisco del Cerro Velázquez y Francisco Javier Ramón Cano.

Página 3 de 13 
En la tabla 1, se presenta el ejemplo de una cualificación profesional de la familia profesional de Servicios Socioculturales y a la Comunidad de nivel 2 con la competencia general, sus unidades de competencia y, ocupaciones y puestos de trabajo asimilados. En la siguiente tabla 2 , se muestra el nivel formativo y entorno profesional de la misma cualificación profesional.

Tabla 1. Descripción de una cualificación profesional (SSC089-2)

\begin{tabular}{|c|c|}
\hline \multicolumn{2}{|r|}{ Cualificación profesional } \\
\hline $\begin{array}{c}\text { Datos de } \\
\text { identificación }\end{array}$ & $\begin{array}{l}\text { Familia profesional: Servicios socioculturales y a la comunidad } \\
\text { Cualificación profesional: SSC089_2 "Atención sociosanitaria a personas en el domicilio". } \\
\text { Nivel: } 2 \text {. }\end{array}$ \\
\hline $\begin{array}{c}\text { Competencia } \\
\text { General }\end{array}$ & $\begin{array}{l}\text { Ayudar en el ámbito socio- sanitario en el domicilio a personas con especiales necesidades de salud } \\
\text { física, psíquica e social, aplicando las estrategias y procedimientos más adecuados para mantener y } \\
\text { mejorar su autonomía personal y sus relaciones con el entorno. }\end{array}$ \\
\hline $\begin{array}{l}\text { Unidades de } \\
\text { competencia }\end{array}$ & $\begin{array}{l}\text { - Desarrollar intervenciones de atención física domiciliaria dirigidas a personas con necesidades de } \\
\text { atención socio- sanitaria (UC0249_2) } \\
\text { - Desarrollar intervenciones de atención psicosocial domiciliaria dirigidas a personas con } \\
\text { necesidades de atención socio- sanitaria (UC0250_2) } \\
\text { - Desarrollar las actividades relacionadas con la gestión y funcionamiento de la unidad convivencial } \\
\text { (UC0251_2) }\end{array}$ \\
\hline $\begin{array}{l}\text { Ocupaciones y } \\
\text { puestos de } \\
\text { trabajo } \\
\text { asimilados }\end{array}$ & $\begin{array}{l}\text { Ocupaciones relacionadas según CNO-2011: No se establecen. } \\
\text { Otras ocupaciones relacionadas: Cuidador de personas mayores, cuidador de discapacitados, } \\
\text { cuidador de convalecientes en el domicilio. }\end{array}$ \\
\hline
\end{tabular}

Tabla 2. Nivel formativo y entorno profesional de una cualificación profesional (SSC089-2)

\begin{tabular}{|c|l|}
\hline \multicolumn{1}{|c|}{ Nivel formativo y entorno profesional de la cualificación profesional } \\
Formativo & Educación Secundaria Obligatoria o Ciclo formativo de grado medio. \\
\hline \multirow{5}{*}{$\begin{array}{c}\text { Entorno } \\
\text { Profesional }\end{array}$} & $\begin{array}{l}\text { Ámbito profesional: } \\
\text { En general, podrá ejercer su actividad en la organización, ejecución y control de las actividades de } \\
\text { atención socio-sanitaria directa a las personas y a su entorno en el domicilio. En concreto podrá ser } \\
\text { contratado por: Administraciones públicas, fundamentalmente municipales y autonómicas, } \\
\text { encargadas de la gestión y prestación de servicios domiciliarios de atención a personas } \\
\text { usuario, como si son responsables de la gestión de servicios o programas de atención domiciliaria de } \\
\text { titularidad pública. Como autónomo, ofertando sus servicios directamente a unidades convivenciales } \\
\text { demandantes o creando su propia empresa de servicios domiciliarios, asociado con otros técnicos. }\end{array}$ \\
\cline { 2 - 3 } & $\begin{array}{l}\text { Sectores productivos: } \\
\text { Podrá ejercer sus funciones en los siguientes centros o equipamientos: Domicilios particulares. Pisos } \\
\text { tutelados. Servicio de atención domiciliaria. }\end{array}$ \\
\hline
\end{tabular}

Según Homs (2008) el sistema español de formación profesional está concebido en sus textos fundamentales desde una visión sistémica, comprensiva de todos los tipos de formación y estrechamente ligada al mercado de trabajo. Efectivamente, el SNCyFP está constituido por dos subsistemas: el de Formación Profesional Inicial (sistema educativo-títulos) y el de Formación Profesional para el Empleo (trabajo y 
empleo-certificados de profesionalidad), ambos determinados en sus enseñanzas por el CNCP.

Las cualificaciones profesionales recogidas en el CNCP vienen organizadas en 26 familias profesionales establecidas al efecto, indicándose para cada cualificación el nivel que le corresponde de acuerdo al grado preciso de conocimiento, iniciativa, autonomía y responsabilidad para realizar dicha actividad laboral. Además, como se ha indicado anteriormente, constituyen el referente para la elaboración de los títulos de formación profesional y los certificados de profesionalidad, que junto al procedimiento establecido para el reconocimiento por experiencia laboral de la competencia profesional, suponen al ciudadano las tres vías de acreditación de competencias de la forma siguiente:

- La primera vía de acreditación corresponde a los títulos de formación profesional del sistema educativo establecidos como un conjunto de enseñanzas normalizadas por la Administración Educativa que capacitan para el desempeño de diversas profesiones técnicas. Los títulos son de carácter oficial y abracan desde la Formación Profesional Básica cuya enseñanza es de oferta obligatoria, los títulos de Técnico (ciclos formativos de grado medio) que forma parte de la educación secundaria post-obligatoria y los de Técnico Superior (ciclos formativos de grado superior) que forma parte de la educación superior acreditando los niveles de las distintas cualificaciones profesionales, respectivamente, de forma escalonada 1, 2, 3 (Real Decreto 1147/2011, de 29 de julio). Los títulos actualmente existentes se pueden consultar en la plataforma on-line de divulgación de la Formación Profesional en España (www.todofp.es), en la sección qué, cómo y dónde estudiar.

- La segunda vía de acreditación reside en la formación profesional para el empleo a través de la parcelación de formación que se ciñe a certificados de profesionalidad como acreditación oficial (Real Decreto 34/2008, de 18 de enero), cuya competencia es de la Administración Laboral encargada de acreditar de forma oficial las cualificaciones profesionales en el ámbito de la Administración Laboral. Se trata de cursos de formación dirigidos prioritariamente a los trabajadores tanto desempleados como ocupados cuyos programas formativos tienen como referencia el $\mathrm{CNCP}$, principal diferencia sobre el resto de los cursos de formación que oferta y acreditan la capacitación para el desarrollo de una actividad laboral con significación para el empleo. Los certificados de profesionalidad por familia profesional se pueden consultar en la sección de formación de la página web https://www.sepe.es/ del Servicio Público de Empleo Estatal.

- La tercera vía es conocida por la abreviatura PREAR (Procedimiento de reconocimiento, evaluación, acreditación y registro de la competencia profesional), se trata de un conjunto de actuaciones dirigidas a reconocer, evaluar y acreditar la competencia profesional que se ha adquirido a través de la experiencia laboral que acredite el candidato así como las vías no formales de formación que disponga (Real Decreto 1224/2009).

Por tanto, el denominador común en cuanto a la acreditación de competencias son las cualificaciones profesionales que suponen el elemento integrador que permite establecer las conexiones oportunas entre el subsistema de Formación Profesional para el Empleo y

Formación Profesional: Requerimientos profesionales y ocupaciones laborales de la CNO-11. Francisco del Cerro Velázquez y Francisco Javier Ramón Cano.

Página 5 de 13 
la Formación Profesional del Sistema Educativo, abriendo así la posibilidad de capitalizar la formación adquirida en un subsistema, en el otro, de la forma que corresponda.

En la figura 1 siguiente podemos ver un esquema resumen de todo lo comentado respecto a las cualificaciones profesionales.

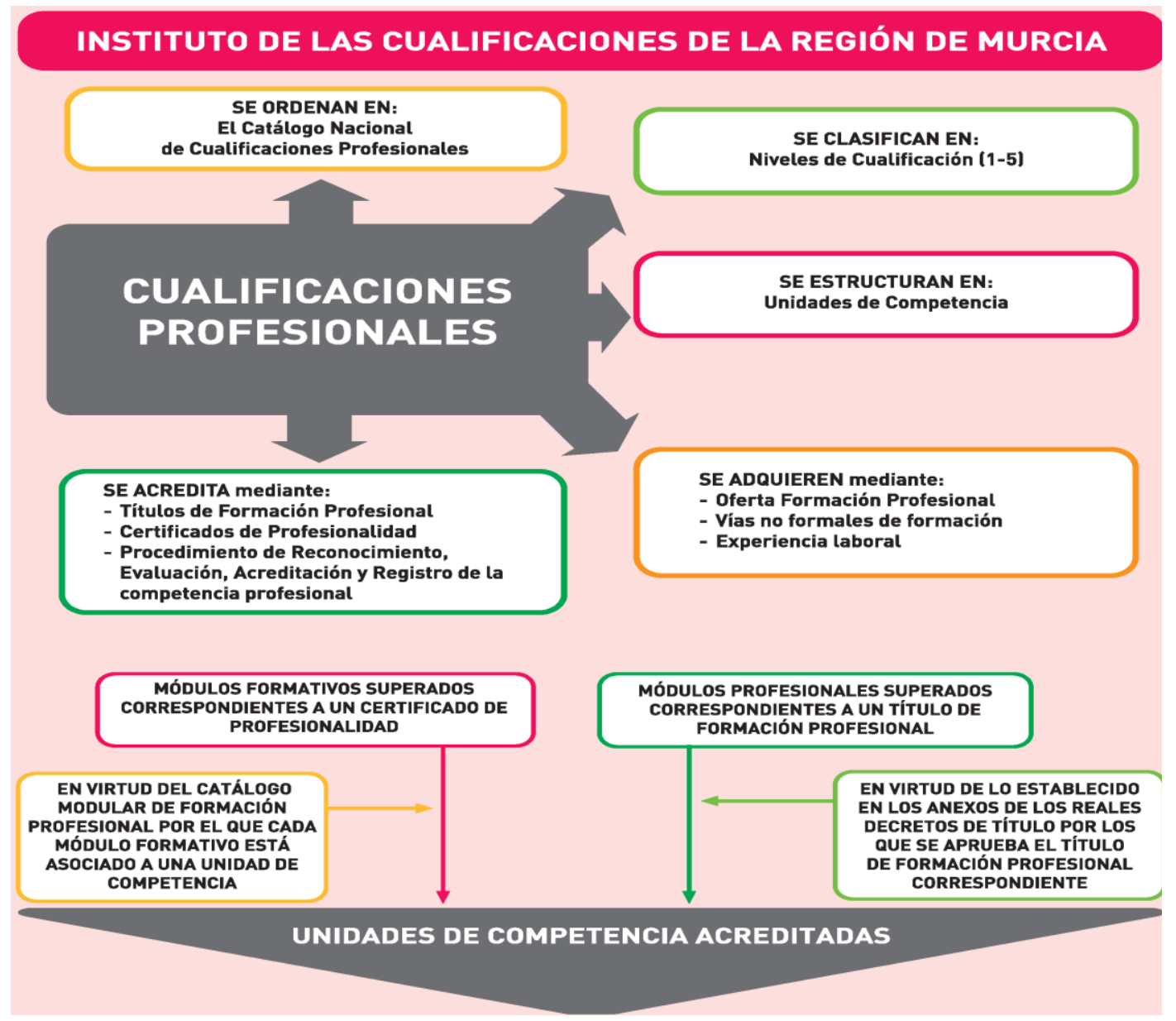

Figura 1. Estructura de las cualificaciones profesionales

Fuente: Consejería competente en materia de Educación de la Región de Murcia

Por otro lado, las ocupaciones laborales más habituales del mercado de trabajo de un país se recogen de forma sistemática en el documento Clasificación Ocupacional, cuyos criterios de elaboración suelen ser, frecuentemente, los sectores o ramas de producción y las exigencias formativas o cualificación profesional.

En España, se encuentra actualmente vigente la Clasificación Nacional de Ocupaciones (CNO-11) que es un sistema de codificación que refleja de manera lo más fidedigna posible la realidad nacional en torno a las ocupaciones $\mathrm{y}$, al mismo tiempo, tiene como referencia la Clasificación Internacional Uniforme de Ocupaciones (CIUO) elaborada por la Oficina Internacional del Trabajo (OIT), por lo que dicha clasificación también es homologable a las clasificaciones de países de nuestro entorno.

Formación Profesional: Requerimientos profesionales y ocupaciones laborales de la CNO-11. Francisco del Cerro Velázquez y Francisco Javier Ramón Cano.

Página 6 de 13 
La CNO-11 es una clasificación llevada a cabo por el Instituto Nacional de Estadística (INE) que tiene por objeto acotar los diferentes ámbitos ocupacionales con el fin de poder garantizar el tratamiento uniforme de datos estadísticos con un montante de 502 ocupaciones codificadas con cuatro dígitos (grupos primarios). La variable "ocupación laboral" ha cobrado importancia desde el punto de vista estadístico, al considerarse como nuclear en los sistemas de estadística socio-demográficos, lo que obliga a mantener siempre actualizada dicha variable (Real Decreto 1591/2010).

Motivados por la falta de la información de las ocupaciones y puestos de trabajos más relevantes asociados a ocupaciones laborales tipificadas en la CNO-11 en los Reales Decretos de creación de los títulos de formación profesional, se plantea la necesidad de plasmar una metodología que permita trasladar la información de dicha variable ocupación laboral a las enseñanzas de formación profesional, los títulos y certificados de profesionalidad. Asimismo, nos interesa vincular la información sobre las condiciones psicofísicas ligadas a situaciones de seguridad o salud derivadas de la actividad laboral y las circunstancias específicas del ambiente de trabajo de las ocupaciones laborales con los títulos de formación profesional.

\section{Objetivos del estudio}

Los objetivos de este estudio han sido:

- Proporcionar una relación entre las enseñanzas de formación profesional y las ocupaciones laborales más frecuentes en el mercado laboral español tipificadas en la CNO-11.

- Identificar en los títulos de formación profesional las condiciones psicofísicas ligadas a situaciones de seguridad o salud de acuerdo al perfil profesional asociado al mismo (requerimientos profesionales).

\section{Metodología}

La metodología empleada en este trabajo se ha basado en el análisis documental a través de catalogación analítica de las siguientes fuentes de información:

$1^{\circ}$. La Guía de Valoración Profesional del Instituto Nacional de la Seguridad Social ( $3^{\mathrm{a}}$ edición año 2014) compuesta por un conjunto de fichas, una por cada ocupación laboral de la CNO-2011, con tres bloques de contenidos diferenciados:

a) Identificación de la profesión y descripción de competencias y tareas donde se incluye el código, denominación y referencia normativa (RD) de las cualificaciones profesionales del CNCP equivalentes o relacionadas con la ocupación laboral definida.

b) Posibles riesgos y circunstancias específicas.

c) Requerimientos profesionales.

$2^{\circ}$. Las cualificaciones profesionales de los títulos de formación profesional de la Ley Orgánica 2/2006, de 3 de mayo, de Educación como instrumento de acreditación oficial de las mismas en el ámbito de la Administración educativa, teniendo en cuenta que para

Formación Profesional: Requerimientos profesionales y ocupaciones laborales de la CNO-11. Francisco del Cerro Velázquez y Francisco Javier Ramón Cano.

Página 7 de 13 
cada uno de los títulos se incorpora, al menos, una cualificación profesional completa y albergando en muchos casos otras cualificaciones profesionales de carácter incompleto.

$3^{\circ}$. Las cualificaciones profesionales de los certificados de profesionalidad del Real Decreto 34/2008, de 18 de enero como instrumento de acreditación oficial de las mismas en el ámbito de la Administración laboral, regulados de forma inicial por dicho Real Decreto (2008) y posteriores modificaciones por el Real Decreto (2010) y Real Decreto (2013), que alojan en sus desarrollos de creación al menos una cualificación profesional completa.

Como muestra de datos se han tomado las publicaciones oficiales en torno al CNCP de fecha límite hasta junio del año 2016 relativas a las cualificaciones profesionales como elemento de referencia para elaborar el perfil profesional asociado un nivel (1,2 o 3$)$ y encuadrada en una familia profesional, los títulos de formación profesional de la enseñanza reglada del sistema educativo, los certificados de profesionalidad de la enseñanza del sistema de formación para el empleo y las ocupaciones laborales según la CNO-11.

La estructura organizativa y metodológica empleada ha sido la siguiente:

a) La primera actuación ha consistido en recabar las cualificaciones profesionales del CNCP para cada una de las familias profesionales.

b) A partir de los datos anteriores, se procede a construir una matriz inicial donde se asocia cada cualificación profesional con los diferentes títulos y certificados de profesionalidad $\mathrm{y}$, a su vez con cada una de las 26 familias profesionales correspondientes procediendo a su clasificación en los distintos niveles 1,2 y 3 que pueden presentarse en el ámbito de la formación profesional.

c) Con los datos anteriores, se procede a realizar un trabajo de investigación documental independiente para cada ocupación laboral de la CNO-11 de la forma siguiente:

$1^{\circ}$ De acuerdo a la información del documento de la Guía de Valoración Profesional se obtiene por cada ocupación laboral las cualificaciones profesionales correspondientes a la misma.

$2^{\circ}$ Una vez obtenidas las cualificaciones profesionales asociadas a la ocupación laboral por la guía de valoración profesional se procedía a confrontar dichas cualificaciones con las acreditadas en las enseñanzas de formación profesional (tanto por los títulos de formación profesional como por los certificados de profesionalidad). De esta manera, se ha conseguido ir asociando las ocupaciones laborales con los títulos de formación profesional y certificados de profesionalidad a través del nexo común que las une, la cualificación profesional. $3^{\circ}$ Otra labor metodológica ha consistido en ligar los requerimientos profesionales que venían registrados en las ocupaciones laborales con los títulos de formación profesional. Al estar las ocupaciones laborales relacionadas con los títulos de formación profesional, por la propiedad asociativa se permitía enlazar los requerimientos profesionales con los títulos de formación profesional.

d) Tratamiento y registro de los datos anteriores a través de una hoja de cálculo Excel 2007.

e) Interpretación de resultados y elaboración de conclusiones.

Formación Profesional: Requerimientos profesionales y ocupaciones laborales de la CNO-11. Francisco del Cerro Velázquez y Francisco Javier Ramón Cano.

Página 8 de 13 
La casuística ha sido variada, de forma que se han encontrado ocupaciones laborales que se ajustaban totalmente con el contenido de una cualificación profesional o varias cualificaciones profesionales correspondientes al mismo título de FP, otras se relacionaban con una o más cualificaciones profesionales que pertenecían a diferentes títulos. En determinados casos, se han encontrado títulos de FP que tenían las cualificaciones profesionales pero de forma incompleta por lo que se ha hecho constar en la relación que dicha formación reglada es insatisfactoria a nivel teórico para afrontar con éxito las exigencias de la ocupación laboral.

Las ocupaciones laborales asociadas a estudios de grado universitario o superiores no han podido ser vinculadas al no disponer en el catálogo de cualificaciones profesionales de nivel 4 y 5. En determinados casos no ha sido posible de establecer una conexión al no disponer de títulos de FP que alberguen las cualificaciones profesionales.

\section{Resultados}

Mediante el planteamiento ofrecido, utilizando las cualificaciones profesionales como denominador común hasta el tercer nivel de los cinco existentes, se ha conseguido relacionar con títulos de Formación Profesional del sistema educativo unas 225 ocupaciones laborales de las 502 ocupaciones incluidas en la CNO-11, estando implicadas todas las familias profesionales, a excepción de la de Actividades Físicas y Deportivas debido a que en la fecha de partida del estudio no se disponía de títulos de formación profesional de esta familia profesional que fueran determinados por cualificaciones profesionales. En cuanto a certificados de profesionalidad se ha logrado asociar hasta 249 ocupaciones laborales, en este caso, abarcando la totalidad de las familias profesionales existentes.

En cuanto a las condiciones psicofísicas ligadas a situaciones de seguridad o salud asociadas al perfil profesional del ciclo formativo de formación profesional (requerimientos profesionales) se han logrado identificar las mismas a nivel teórico en un total de 131 títulos de Formación Profesional de los 163 títulos que a nivel nacional se disponen en el 2018; proporcionándose para cada una de las ocupaciones laborales principales del título los posibles riesgos derivados de la actividad laboral y las circunstancias específicas del ambiente de trabajo que puedan incidir en la capacidad laboral de los trabajadores, en base a las pautas ligadas a la información del Instituto Nacional de Seguridad e Higiene en el Trabajo.

La relación entre las ocupaciones laborales de la CNO-2011 con los títulos de formación profesional derivados de la Ley Orgánica 2/2006, de 3 de mayo, de Educación y los certificados de profesionalidad, por la extensión de los resultados conseguidos, se pueden consultar en la herramienta interactiva alojada en la página web http://www.um.es/didac_tecyfp/fp/ocupacionesfp/ creada al efecto por León Morales (2018). En dicha página web también es posible descargar el documento de las condiciones psicofísicas ligadas a situaciones de seguridad o salud de los títulos de formación profesional en función de las ocupaciones más relevantes que se han adscrito a los mismos desarrollado en Yanes Marcelino (2018).

Formación Profesional: Requerimientos profesionales y ocupaciones laborales de la CNO-11. Francisco del Cerro Velázquez y Francisco Javier Ramón Cano.

Página 9 de 13 


\section{Conclusiones}

En este trabajo se ha conseguido establecer un particular punto de vista de la información del binomio variable ocupación laboral estandarizado en la CNO-11 y los títulos de formación profesional $\mathrm{y}$, por ende, se abre la posibilidad de tratamiento estadístico de dichos datos, lo que permite que sean comparables a nivel internacional. En este sentido, aunque no podemos afirmar que sea novedosa la relación establecida de las ocupaciones laborales de la CNO-11 con los certificados de profesionalidad, ya que en el portal web del Servicio Público de Empleo Estatal se encuentra dicha información desarrollada a través de fichas con las ocupaciones estandarizadas y puestos de trabajo relacionados para cada certificado, este artículo aporta nuevos nutrientes a la información referida para alcanzar la competencia del ciudadano acorde a las exigencias de la profesión deseada, con una ventaja competitiva innegable desde el punto de vista de la orientación basado en la exposición de forma simultánea del abanico de posibilidades en formación en educación (títulos de formación profesional) y en empleo (certificados de profesionalidad).

La metodología empleada permite obtener a nivel teórico una relación inequívoca y estandarizada entre ocupación laboral y formación profesional y, además, permite seguir avanzando en establecer relaciones entre las ocupaciones laborales que se vayan desarrollando en la CNO-11 y las nuevas enseñanzas de formación profesional.

En cuanto a los resultados obtenidos de las condiciones psicofísicas ligadas a situaciones de seguridad o salud asociadas al perfil profesional (requerimientos profesionales) del ciclo de la formación profesional, connotar que somos conscientes que los mismos no están exentos de controversia tanto a la hora de precisar dicha relación tanto conceptual como de normalización de los niveles de cualificación profesional que requieren las ocupaciones laborales, presentándose en determinados títulos de las familias profesionales una información poco exhaustiva, en cualquier caso, dichos resultados no pretenden interferir en la vocación profesional del ciudadano.

Con fines de mejora de la orientación educativa y profesional en España, el trabajo está abierto a su enriquecimiento en un marco de coordinación de todos los actores de las Administraciones educativas y laborales, de la administración local, de los interlocutores sociales, y de cualquier otro organismo o entidad que preste servicios de orientación profesional.

Presentación del artículo: 15 de mayo de 2018

Fecha de aprobación: 9 de julio de 2018

Fecha de publicación: 31 de octubre de 2018

Del Cerro Velázquez, F. y Ramón Cano, F.J. (2018). Formación Profesional: Requerimientos profesionales y ocupaciones laborales de la CNO-11. RED. Revista de Educación a Distancia, 58. Consultado el (dd/mm/aaaa) en http://www.um.es/ead/red/

Formación Profesional: Requerimientos profesionales y ocupaciones laborales de la CNO-11. Francisco del Cerro Velázquez y Francisco Javier Ramón Cano.

Página 10 de 13 


\section{Financiación}

El presente trabajo es una continuación de otro estudio sobre la empleabilidad de los recién titulados de la formación profesional cuyos los datos fueron cedidos por el Servicio de Formación Profesional de la Región de Murcia en el proyecto de investigación "Inserción laboral de titulados de Formación Profesional en la Región de Murcia -Curso 2013/2014“, con el objetivo de poder avanzar en el estudio del binomio inserción laboral-formación profesional. La financiación inicial correspondió a la Dirección General de Formación Profesional de la Región de Murcia.

\section{Bibliografía}

Amor Bravo, E. (2014): Las cualificaciones profesionales: un reto para los sistemas de educación y formación de la sociedad española. Revista del Ministerio de Trabajo e Inmigración. 81, pp. 89-103.

Cedefop (2015). Mercado de trabajo global, formación profesional global, Luxemburgo, Oficina de Publicaciones Oficiales de las Comunidades Europeas, 2015. (Cedefop reference series).

Clasificación Nacional de Ocupaciones 2011 (CNO-11). Descripción [Internet]. Instituto Nacional de Estadística. Recuperado el 2 de septiembre de 2016, de: http://www.ine.es/jaxi/menu.do?type=pcaxis \&path=/t40/cno11\&file=inebase

Del Cerro Velázquez, F. y Ramón Cano, F.J. (2017). Compromiso y empleabilidad de los recién titulados de formación profesional. Conclusiones para un rediseño de la modalidad formativa. RED. Revista de Educación a Distancia, 54. Consultado el (19/07/2017) en http://www.um.es/ead/red/

Echeverría, B. (coord.) (2008). Orientación Profesional. Barcelona, Editorial UOC.

Guía de valoración profesional (2014). Instituto Nacional de Seguridad e Higiene en el Trabajo (INSHT), España. ( $3^{a}$ edición). Recuperado el 2 de septiembre de 2016, de: http://www.seg-social.es/prdi00/groups/public/documents/binario/198948.pdf

Homs, O. (2008): La formación profesional en España. Hacia la sociedad del conocimiento. Fundación "la Caixa" (Colección Estudios Sociales, no 25), Barcelona.

León Morales, D. (2018). Herramienta interactiva para relacionar las ocupaciones laborales de la CNO-11 con la formación profesional (Trabajo fin de máster). Universidad de Murcia, Facultad de Educación.

Formación Profesional: Requerimientos profesionales y ocupaciones laborales de la CNO-11. Francisco del Cerro Velázquez y Francisco Javier Ramón Cano. 
Ley Orgánica 5/2002, de 19 de junio, de las Cualificaciones y de la Formación Profesional. Boletín Oficial del Estado, 147, 20 de junio de 2002.

Ley Orgánica 2/2006, de 3 de mayo, de Educación. Boletín Oficial del Estado, 106, de 4 de mayo de 2006, pp 17158-17207.

Medina, O. y Sanz, F. (2009). Los sistemas de reconocimiento y acreditación de los aprendizajes no formales e informales: referencias históricas, funciones socioeducativas y perspectiva teórica. Revista de Educación, 348, 253-281.

Mira Lema, J.L. (2003). El reto de la Formación Profesional Bordón. Revista de pedagogía, Vol. 55, No 3, 335-342.

OCDE (2014). Panorama de la educación. Indicadores de la OCDE 2014. Informe español. Disponible en http://www.mecd.gob.es/dctm/inee/indicadoreseducativos/panorama2014/panorama-de-la-educacion-2014informe-espanol-05-sep.pdf?documentId=0901e72b81a722ac

Planas Domingo, J.A. Nuevas tendencias en la formación profesional. Aula de Secundaria, 4, 29-32.

Pérez Esparrells, C. y Rahona, M. (2009). La calidad de la formación profesional y sus implicaciones para el mercado de trabajo en Los nuevos retos de la Formación Profesional. Revista del Instituto de Estudios Económicos, núm. 2/2009, pp. 71-102.

Real Decreto 1416/2005, de 25 de noviembre, por el que se modifica el Real Decreto 1128/2003, de 5 de septiembre, por el que se regula el catálogo de las cualificaciones profesionales. Boletín Oficial del Estado, 289, 3 de diciembre de 2005, pp 3985439855.

Real Decreto 34/2008, de 18 de enero, por el que se regulan los certificados de profesionalidad. Boletín Oficial del Estado, 27, de 31 de enero de 2008.

Real Decreto 1224/2009, de 17 de julio, de reconocimiento de las competencias profesionales adquiridas por experiencia laboral. Boletín Oficial del Estado, 205, 25 de agosto de 2009, pp 72704-72727.

Real Decreto 1591/2010, de 26 de noviembre, por el que se aprueba la Clasificación Nacional de Ocupaciones 2011. Boletín Oficial del Estado, 306, 17 de diciembre de 2010 .

Formación Profesional: Requerimientos profesionales y ocupaciones laborales de la CNO-11. Francisco del Cerro Velázquez y Francisco Javier Ramón Cano. 
Real Decreto 1675/2010, de 10 de diciembre, por el que se modifica el Real Decreto $34 / 2008$, de 18 de enero, por el que se regulan los certificados de profesionalidad y los reales decretos por los que se establecen certificados de profesionalidad dictados en su aplicación. Boletín Oficial del Estado, 318, 31 de diciembre de 2010, pp 109381-109386.

Real Decreto 1147/2011, de 29 de julio, por el que se establece la ordenación general de la formación profesional del sistema educativo. Boletín Oficial del Estado, 182, 30 de julio de 2011, pp 86766-86800.

Real Decreto 189/2013, de 15 de marzo, por el que se modifica el Real Decreto 34/2008, de 18 de enero, por el que se regulan los certificados de profesionalidad y los reales decretos por los que se establecen certificados de profesionalidad dictados en su aplicación. Boletín Oficial del Estado, 69, 21 de marzo de 2013, pp 22411-22434.

Sepe.es, plataforma on-line de divulgación del Servicio Público de Empleo Estatal. Certificados de profesionalidad: (Fecha de acceso: julio de 2018)

Sparreboom, T. (2013, 4 de febrero). El desajuste entre oferta y demanda de calificaciones afecta la creación de empleo. Recuperado de http://www.ilo.org/

Todofp.es, plataforma on-line de divulgación de la Formación Profesional en España. Qué, cómo y dónde estudiar: (Fecha de acceso: julio de 2018).

Yanes Marcelino, J. (2018). Análisis de la formación profesional desde el punto de vista de los futuros requerimientos profesionales (Trabajo fin de máster). Universidad de Murcia, Facultad de Educación. 